\title{
SOME FIXED POINT THEOREMS FOR COMPOSITES OF ACYCLIC MAPS
}

\author{
SEHIE PARK, S. P. SINGH, AND BRUCE WATSON
}

(Communicated by Palle E. T. Jorgensen)

\begin{abstract}
We obtain fixed point theorems for a new class of multifunctions containing compact composites of acyclic maps defined on a convex subset of a locally convex Hausdorff topological vector space. Our new results are applied to approximatively compact, convex sets or to Banach spaces with the Oshman property.
\end{abstract}

\section{INTRODUCTION}

Recently there have appeared a number of new results on fixed points of so-called Kakutani factorizable multifunctions or composites of acyclic multifunctions defined on convex subsets of topological vector spaces; for example, see $[1,2,3,13,14,18]$. Especially in [14] Lassonde considered a class $\mathbb{K}_{c}^{+}$of multifunctions containing Kakutani factorizable multifunctions and obtained very general results.

In the present paper we replace the convexity of the functional values by acyclicity and, motivated by [14], consider a class $\mathbb{V}_{c}^{+}$of multifunctions properly containing $\mathbb{K}_{c}^{+}$and composites of acyclic maps. First we obtain generalizations of a coincidence theorem of Granas and Liu [10] and a fixed point theorem of Lassonde [14, 12]. Then we apply these theorems to generalize results of Reich [20] on approximatively compact sets or on Banach spaces with the Oshman property.

\section{Preliminaries}

A multifunction $F: X \rightarrow 2^{Y}$ is a function with set values $F x \subset Y$ for each $x \in X$. The set $\{(x, y): y \in F x\}$ is called either the graph of $F$ or, simply, $F$. So $(x, y) \in F$ if and only if $y \in F x$. For any $C \subset X$ let $F(C)=\bigcup\{F x: x \in C\}$. For any $B \subset Y$ let $F^{-}(B)=\{x \in X: F x \cap B \neq \varnothing\}$. If $B$ is a singleton $\{y\}$ in $Y$ then $F^{-}(B)$ is called a fiber denoted $F^{-} y$.

Received by the editors October 6, 1992 and, in revised form, November 9, 1992.

1991 Mathematics Subject Classification. Primary 47H10; Secondary 54H25, 55M20.

Key words and phrases. Acyclic map, approximatively compact, Kakutani factorizable multifunction, coincidence point.

The first author was partially supported by KOSEF-GARC in 1992 and was a Visiting Research Professor at Memorial University when this work was completed. 
In this paper a convex space $C$ is a nonempty convex set in a vector space with any topology that induces the Euclidean topology on the convex hulls of its finite subsets (see [12]). Such convex hulls are called polytopes.

For topological spaces $X$ and $Y$ a multifunction $F: X \rightarrow 2^{Y}$ is upper semicontinuous (u.s.c.) if, for each closed set $B \subset Y, F^{-}(B)$ is closed in $X$. $F$ is lower semicontinuous (l.s.c.) if, for each open set $B \subset Y, F^{-}(B)$ is open in $X . F$ is continuous if $F$ is both u.s.c. and l.s.c. $F$ is compact if $F(X)$ is contained in a compact subset of $Y$. A set $K \subset X$ is called $\sigma$-compact if $K$ is the countable union of compact sets. A nonempty topological space is acyclic if all its reduced Čech homology groups over rationals vanish.

For a given class $\mathbb{L}$ of multifunctions define

$$
\mathbb{L}(X, Y)=\left\{T: X \rightarrow 2^{Y} \mid T \in \mathbb{L}\right\}, \quad \mathbb{L}_{c}=\left\{T=T_{m} T_{m-1} \cdots T_{1} \mid T_{i} \in \mathbb{L}\right\} .
$$

Using the above notation we have the following definitions:

(1) If $f$ is continuous and single-valued, we write $f \in \mathbb{C}(X, Y)$.

(2) We say that $F$ is a Kakutani map and write $F \in \mathbb{K}(X, Y)$ if $Y$ is a convex space and $F$ is u.s.c. with nonempty compact convex values.

(3) $F$ is an acyclic map, written $F \in \mathbb{V}(X, Y)$, if $F$ is u.s.c. with compact acyclic values.

(4) $F \in \mathbb{K}^{+}(X, Y)$ (resp. $\mathbb{V}^{+}(X, Y)$ ) if for any $\sigma$-compact subset $K$ of $X$ there is a $\Gamma \in \mathbb{K}(K, Y)(\mathbb{V}(K, Y))$ such that $\Gamma x \subset F x$ for each $x \in K$.

(5) $F \in \mathbb{K}_{c}^{+}(X, Y)$ (resp. $\mathbb{V}_{c}^{+}(X, Y)$ ) if for any $\sigma$-compact subset $K$ of $X$ there is a $\Gamma \in \mathbb{K}_{c}(K, Y)\left(\mathbb{V}_{c}(K, Y)\right)$ such that $\Gamma x \subset F x$ for each $x \in K$.

(6) $F \in \mathbb{M}(X, Y)$ if $F x$ is convex for all $x \in X$ and $F^{-} y$ is open for all $y \in Y$.

(7) $F \in \mathbb{T}(X, Y)$ if $F x$ is convex and $\bigcup_{V \in \mathscr{V}(x)}\left(\bigcap_{x^{\prime} \in V} F x^{\prime}\right) \neq \varnothing$ for all $x \in X$, where $\mathscr{V}(x)$ is a neighborhood base of $x$ in $X$ (see Lassonde [14]).

It is known that $\mathbb{K}_{c}^{+}$contains $\mathbb{C}, \mathbb{M}, \mathbb{K}, \mathbb{K}_{c}$, and $\mathbb{T}$ (see [14]). Moreover, it is clear that $\mathbb{V}_{c}^{+}$includes $\mathbb{V}_{c}$ and $\mathbb{K}_{c}^{+}$.

Let $C$ be a subset of a topological vector space $E$, and suppose $x \in E$. The inward set $I_{C}(x)$ and outward set $O_{C}(x)$ of $C$ at $x$ are defined, respectively, by

$$
\begin{aligned}
& I_{C}(x)=\{x+r(y-x): y \in C, r \geq 0\}, \\
& O_{C}(x)=\{x+r(y-x): y \in C, r \leq 0\} .
\end{aligned}
$$

Their closures will be denoted by $\overline{I_{C}(x)}$ and $\overline{O_{C}(x)}$.

For the remaining definitions in this section assume that $C$ is a nonempty subset of a locally convex Hausdorff topological vector space $E$ and that $p$ is a continuous seminorm on $E$.

For $y \in E$ define $d_{p}(y, C)=\inf \{p(y-x): x \in C\}$ and $Q_{p}(y)=\{x \in$ $\left.C: p(y-x)=d_{p}(y, C)\right\} . C$ is called approximatively compact (with respect to $p$ ) if for each $y \in E$ every net $\left\{x_{\alpha}: \alpha \in \Lambda\right\} \subset C$ such that $p\left(y-x_{\alpha}\right) \rightarrow d_{p}(y, C)$ has a subnet that converges to an element of $C$.

We list some boundary conditions for a multifunction $F: C \rightarrow 2^{E}$. We use $\partial C$ to denote the boundary of $C$, and each condition must hold for all 
$x \in \partial C \backslash F x:$

(0) For each $y \in F x$ and each continuous seminorm $p, p(y-x)>0$ implies $p(y-x)>p(y-z)$ for some $z \in \overline{I_{C}(x)}$.

(i) For each $y \in F x$ there exists a number $\lambda$ (real or complex, depending on whether $E$ is real or complex) such that

$$
|\lambda|<1 \text { and } \lambda x+(1-\lambda) y \in \overline{I_{C}(x)} \text {. }
$$

(ii) $F x \subset \overline{I_{C}(x)}$.

(iii) For each $y \in F x$ there exists a $\lambda$ (as above) such that

$$
|\lambda|<1 \text { and } \lambda x+(1-\lambda) y \in C \text {. }
$$

(iv) $F x \subset I F_{C}(x):=\left\{x+c(u-x): u \in C, \operatorname{Re}(c)>\frac{1}{2}\right\}$.

(v) $F x \subset C$.

(vi) $F(C) \subset C$.

It is well known that (vi) $\Rightarrow(\mathrm{v}) \Rightarrow$ (iv) $\Rightarrow$ (ii) $\Rightarrow$ (i) and that (iv) $\Leftrightarrow$ (iii) $\Rightarrow$ (i) $\Rightarrow(0)$.

Moreover, each of (0), (i), (ii), and (iv) has a corresponding outward boundary condition.

Note that for a convex-valued multifunction we can consider more general conditions than some of (i)-(vi) (see Park [17]).

\section{Main Results}

We need the following lemma in our subsequent work.

Lemma 1. Let $P$ be a polytope. If $F \in \mathbb{V}_{c}(P, P)$ then $F$ has a fixed point.

This fact is quite well known in the Lefschetz fixed point theory. For details we refer the reader to $[19,8,9]$.

Recently there have appeared several interesting coincidence theorems. We give the following, which unifies several known results. We use a partition of unity argument in the proof.

Theorem 1. Let $X$ be a convex space, $Y$ a Hausdorff space, and $F, G: X \rightarrow 2^{Y}$ multifunctions satisfying.

(1) $F \in \mathbb{V}_{c}^{+}(X, Y)$ is compact;

(2) for each $y \in F(X), G^{-} y$ is convex; and

(3) $\{$ Int $G x: x \in X\}$ covers $\overline{F(X)}$.

Then $F$ and $G$ have a coincidence point $x_{0} \in X$; that is, $F x_{0} \cap G x_{0} \neq \varnothing$.

Proof. Since $\overline{F(X)}$ is compact and covered by $\{$ Int $G x: x \in X\}$, there is a finite set $N=\left\{x_{1}, x_{2}, \ldots, x_{n}\right\}$ in $X$ such that $\overline{F(X)} \subset \bigcup\{$ Int $G x: x \in N\}$. Let $\left\{\lambda_{1}, \lambda_{2}, \ldots, \lambda_{n}\right\}$ be the partition of unity corresponding to this cover, and let $P$ represent the convex hull of $N$, denoted co $N$. Define $f: \overline{F(X)} \rightarrow P$ by

$$
f y=\sum_{1}^{n} \lambda_{i}(y) x_{i}=\sum_{i \in N_{y}} \lambda_{i}(y) x_{i}
$$

for $y \in \overline{F(X)} \subset Y$, where $i \in N_{y}$ if and only if $\lambda_{i}(y) \neq 0$, which implies that $y \in \operatorname{Int} G x_{i}$. Then, for $i \in N_{y}, x_{i} \in G^{-} y$. Clearly $f$ is continuous and by condition (2) we have $f y \in \operatorname{co}\left\{x_{i}: i \in N_{y}\right\} \subset G^{-} y$ for each $y \in F(X)$. Since 
$P \subset X$ is compact and $F \in \mathbb{V}_{c}^{+}(X, Y)$, there is a $\Gamma \in \mathbb{V}_{c}(P, F(X))$ such that $\Gamma x \subset F x$ for all $x \in P$. Therefore, by Lemma $1, f \Gamma \in \mathbb{V}_{c}(P, P)$ has a fixed point $x_{0} \in P \subset X$. Since $x_{0} \in(f \Gamma) x_{0} \subset(f F) x_{0}$ and $f^{-} x_{0} \subset G x_{0}$, we have

$$
F x_{0} \cap G x_{0} \neq \varnothing \text {. }
$$

This completes the proof.

Particular forms of Theorem 1 were given by Browder [4-6] and Reich [21] with $X=Y$ and $F=1_{X}$, by Granas and Liu [10] with $\mathbb{V}$ instead of $\mathbb{V}_{c}^{+}$, and by Lassonde [13, Theorem 3 for $\mathbb{K}_{c} ; 14$, Theorem 4 for $\left.\mathbb{K}_{c}^{+}\right]$. For related results see [1, Theorems 5.1 and 5.2].

Based on Theorem 1 we give

Theorem 2. Let $X$ and $C$ be nonempty convex subsets of a locally convex Hausdorff topological vector space $E$ and $F \in \mathbb{V}_{c}(X, X+C)$ a compact multifunction. Suppose that one of the following conditions holds.

(1) $X$ is closed and $C$ is compact.

(2) $C$ is closed and $X$ is compact.

(3) $C=\{0\}$.

Then there is an $\hat{x} \in X$ such that $F \hat{x} \cap(\hat{x}+C) \neq \varnothing$.

Proof. Let $V$ be an open convex neighborhood of the origin 0 in $E$, and let $Y$ be a compact set such that $F(X) \subset Y \subset X+C$. Define $G: X \rightarrow 2^{Y}$ by

$$
G x=(x+C+V) \cap Y \text { for } x \in X .
$$

Then each $G x$ is open in $Y$ and $G^{-} y=(y-C-V) \cap X$ is convex for each $y \in Y$. Moreover, since $Y \subset X+C$, for every $y \in Y$ there exists an $x \in X$ such that $y \in x+C+V$; that is, $\{G x: x \in X\}$ covers $Y$. Therefore, by Theorem 1, there exist $x_{V} \in X$ and $y_{V} \in Y$ such that $y_{V} \in F x_{V} \cap G x_{V}$; that is, $y_{V}-x_{V} \in C+V$. Hence for each neighborhood $V$ of 0 in $E$ we get that

$$
(F-i)(X) \cap(C+V) \neq \varnothing,
$$

where $i: X \rightarrow E$ is the inclusion.

Case (1). Because $X$ is closed, so is $(F-i)(X)$. Since $C$ is compact and $E$ is regular, $(*)$ implies $(F-i)(X) \cap C \neq \varnothing$; that is, there is an $\hat{x} \in X$ such that $F \hat{x} \cap(\hat{x}+C) \neq \varnothing$.

Case (2). Since $(F-i)(X)$ is compact and $C$ is closed, the same conclusion follows as in Case (1).

Case (3). Since $F$ is u.s.c., for each neighborhood $V$ of 0 in $E$, there exist $x_{V}, y_{V} \in X$ such that $y_{V} \in F x_{V}$ and $y_{V}-x_{V} \in V$. Since $F(X)$ is relatively compact, we may assume that $y_{V}$ converges to some $\hat{x}$. Then $x_{V}$ also converges to $\hat{x}$. Since the graph of $F$ is closed in $X \times \overline{F(X)}$, we have $\hat{x} \in F \hat{x}$. This completes our proof.

Theorem 2(3) can be stated in the following, seemingly, more general form.

Corollary 1. Let $X$ be a nonempty convex subset of a locally convex Hausdorff topological vector space $E$ and $F \in \mathbb{V}_{c}^{+}(X, X)$. If $F$ is compact, then $F$ has a fixed point. 
Proof. Let $K=\operatorname{co} \overline{F(X)}$. Then $K$ is $\sigma$-compact by Proposition 1(3) of [14]. Since $F \in \mathbb{V}_{c}^{+}(X, K)$ there exists a $\Gamma \in \mathbb{V}_{c}(K, K)$ such that $\Gamma x \subset F x$ for each $x \in K$. Moreover, since $F$ is compact, so is $\Gamma$. Therefore, by Theorem 2(3), $\Gamma$ has a fixed point $x_{0} \in \Gamma x_{0} \subset F x_{0}$. This completes the proof.

Particular forms of Theorem 2 have been given by Lassonde [12, Theorem 1.6 and Corollary 1.18] for $\mathbb{K}$ and Park [16, Theorem 7] for $\mathbb{V}$. Corollary 1 includes Lassonde [14, Théorème 5] for $\mathbb{K}_{c}^{+}$and [13, Theorem 4] for $\mathbb{K}_{c}$, Himmelberg [11, Theorem 2] for $\mathbb{K}$, and many others. Himmelberg's result extends earlier works of Schauder, Mazur, Bohnenblust and Karlin, Hukuhara, Singbal, Tychonoff, Kakutani, Fan, and Glicksberg (for the literature see [16]).

The next result uses an additional lemma given for approximatively compact sets. Recall that a compact set is approximatively compact but that the converse is false.

Lemma 2. Let $C$ be a subset of a Hausdorff topological vector space $E$ and $p$ a continuous seminorm on $E$. If $C$ is approximatively compact (with respect to $p)$, then the metric projection $Q_{p}: E \rightarrow 2^{C}$ is u.s.c.

Lemma 2 is given in [22] and also in [20].

Theorem 3. Let $C$ be a nonempty approximatively compact, convex subset of a locally convex Hausdorff topological vector space $E$, and suppose that $F \in$ $\mathbb{V}_{c}^{+}(C, E)$ is a compact multifunction. Then for each continuous seminorm $p$ on $E$ there exists an $\left(x_{0}, y_{0}\right) \in F$ such that

$$
p\left(x_{0}-y_{0}\right) \leq p\left(x-y_{0}\right) \quad \text { for all } x \in \overline{I_{C}\left(x_{0}\right)} .
$$

Proof. Consider the metric projection $Q_{p}: E \rightarrow 2^{C}$. Clearly $Q_{p}(x)$ is nonempty, compact, and convex for every $x \in E$, and $Q_{p}$ is an u.s.c. multifunction by Lemma 2. Thus $Q_{p} \in \mathbb{K}(E, C) \subset \mathbb{V}^{+}(E, C)$. Since it is clear that $\mathbb{V}_{c}^{+}$ is closed under composition (cf. Lassonde [14, Proposition 2(1)]), we have $Q_{p} F \in \mathbb{V}_{c}^{+}(C, C)$ and $Q_{p} F$ is compact. Therefore, by Corollary $1, Q_{p} F$ has a fixed point; that is, there is a $y_{0} \in F x_{0}$ such that

$$
x_{0} \in Q_{p} y_{0}=\left\{x \in C: p\left(y_{0}-x\right)=d_{p}\left(y_{0}, C\right)\right\} .
$$

Actually, this implies the conclusion by the methods of Park in [15].

Remarks. For $F \in \mathbb{V}_{c}^{+}(C, E)$ define a multifunction $F^{\prime}: C \rightarrow 2^{E}$ by $F^{\prime} x=$ $2 x-F x$ for $x \in C$. If $F^{\prime} \in \mathbb{V}_{c}^{+}(C, E)$, then $\overline{I_{C}\left(x_{0}\right)}$ in the conclusion of Theorem 3 can be replaced by $\overline{O_{C}\left(x_{0}\right)}$.

Note that for $F=f \in \mathbb{C}(C, E)$ Theorem 3 reduces to Reich [20, Corollary 2.2]. For a compact convex subset of a normed vector space $E$, the origins of Theorem 3 go back to the best approximation theorem of Fan [7].

For a continuous multifunction $F: C \rightarrow 2^{E}$ satisfying certain restrictions, some authors obtain the following type of variational inequality:

$$
\text { there exists } x_{0} \in C \text { such that } d_{p}\left(x_{0}, F x_{0}\right)=d_{p}\left(C, F x_{0}\right) \text {. }
$$

It is well known that, in this case, one cannot dispense with the lower semicontinuity of $F$. The following example shows that lower semicontinuity is not necessary in Theorem 3. 
Example. Take $E=\mathbb{R}^{2}$ and $C=[0,1] \times\{0\}$. Suppose $F \in \mathbb{V}(C, E)$ is given by

$$
F(a, 0)= \begin{cases}\{(0,1)\} & \text { if } a \neq 0, \\ {[(0,1),(1,0)] \cup[(-1,0),(0,1)]} & \text { if } a=0,\end{cases}
$$

where $[A, B]$ stands for the closed line segment joining points $A$ and $B$ in the plane. Then $F$ is not lower semicontinuous, but $x_{0}=(0,0)$ and $y_{0}=\left(\frac{1}{2}, \frac{1}{2}\right)$ satisfy the conclusion of Theorem 3.

The following fixed point theorem holds in normed vector spaces.

Corollary 2. Let $C$ be an approximatively compact, convex subset of a normed vector space $E$, and suppose that $F \in \mathbb{V}_{c}^{+}(C, E)$ is compact such that

(0) for all $(x, y) \in F, y \neq x$, we have $\|y-x\|>\|y-z\|$ for some $z \in \overline{I_{C}(x)}$.

Then $F$ has a fixed point.

Proof. By Theorem 3 there exists an $\left(x_{0}, y_{0}\right) \in F$ such that $\left\|x_{0}-y_{0}\right\| \leq\left\|x-y_{0}\right\|$ for all $x \in \overline{I_{C}\left(x_{0}\right)}$. If $x_{0} \neq y_{0}$, then $(0)$ leads to a contradiction. This completes the proof.

Remarks. Suppose $F^{\prime}$ is defined as in the remarks following Theorem 3. If $F^{\prime} \in \mathrm{V}_{c}^{+}(C, E)$, then the outward boundary conditions corresponding to $(0)$, (i), (ii), and (iv) can be used instead of (0) in Corollary 2. In some of these cases we may have some surjectivity; that is, $F(C) \supset C$ (see Park [17]).

If $F \in \mathbb{K}(C, E)$ and $C$ is compact, then Corollary 2 extends Reich [20, Theorem 3.3(a)], who used the boundary condition

$$
F x \cap Q^{-1} x \subset\{x\} \text { for all } x \in C .
$$

Note that $\left(0^{\prime}\right) \Rightarrow(0)$. In fact, if $y \in F x \cap Q^{-1} x$ and $y=x$, then $F$ has a fixed point. If $F x \cap Q^{-1} y=\varnothing$, then any $y \in F x$ satisfies (0).

Recall that a reflexive Banach space has the Oshman property if the metric projection on every closed convex subset is u.s.c.

Theorem 4. Let $C$ be a closed convex subset of a Banach space $E$ with the Oshman property and $F \in \mathbb{V}_{c}^{+}(C, E)$ a compact multifunction. Then there exists $\left(x_{0}, y_{0}\right) \in F$ such that

$$
\left\|x_{0}-y_{0}\right\| \leq\left\|x-y_{0}\right\| \text { for all } x \in \overline{I_{C}\left(x_{0}\right)} .
$$

The proof is like that of Corollary 1, and we omit it here.

Remarks. As in the remarks following Corollary 1, if $F^{\prime} \in \mathbb{V}_{c}^{+}(C, E)$, then the inward set can be replaced by the outward set.

For $F=f \in \mathbb{C}(C, E)$ Theorem 4 reduces to Proposition 2.3 of Reich [20].

Corollary 3. Let $C$ be a closed convex subset of a Banach space $E$ with the Oshman property, and suppose $F \in \mathbb{V}_{c}^{+}(C, E)$ is a compact multifunction satisfying boundary condition ( 0$)$. Then $F$ has a fixed point.

The proof is like that of Corollary 2. 
Remark. Reich [20, Proposition 3.2] obtained Corollary 3 for $F \in \mathbb{K}(C, E)$ satisfying boundary condition (iv). Our proof is different from his. Reich [20, Theorem 3.3(b)] is also a particular form of Corollary 3 for $F \in \mathbb{K}(C, E)$ satisfying condition $\left(0^{\prime}\right)$. Related results for single-valued and set-valued mappings are given in Reich [21].

\section{REFERENCES}

1. H. Ben-El-Mechaiekh, The coincidence problem for compositions of set-valued maps, Bull. Austral. Math. Soc. 41 (1990), 421-434.

2. H. Ben-El-Mechaiekh and P. Deguire, Approximation of non-convex set-valued maps, C. R. Acad. Sci. Paris Sér. I Math. 312 (1991), 379-384.

3. Sér. I Math. 312 (1991), 433-438.

4. F. E. Browder, The fixed point theory of multi-valued mappings in topological vector spaces, Math. Ann. 177 (1968), 283-301.

5. $\_$, On a sharpened form of the Schauder fixed point theorem, Proc. Nat. Acad. Sci. U.S.A. 74 (1977), 4749-4751.

6. __ Coincidence theorems, minimax theorems, and variational inequalities, Contemp. Math., vol. 26, Amer. Math. Soc., Providence, RI, 1984, pp. 67-80.

7. K. Fan, Extensions of two fixed point theorems of F. E. Browder, Math. Z. 112 (1969), 234-240.

8. L. Górniewicz and A. Granas, Some general theorems in coincidence theory. I, J. Math. Pures Appl. (9) 60 (1981), 361-373.

9. L. Górniewicz and A. Granas, Topology of morphisms and fixed point problems for set-valued mappings, Fixed Point Theory and Applications (M. A. Théra and J. B. Baillon, eds.), Longman Sci. Tech., Essex, 1991, pp. 173-191.

10. A. Granas and F.-C. Liu, Coincidences for set-valued maps and minimax inequalities, J. Math. Pures Appl. (9) 65 (1986), 119-148.

11. C. J. Himmelberg, Fixed points of compact multifunctions, J. Math. Anal. Appl. 38 (1972), 205-207.

12. M. Lassonde, On the use of $K K M$-multifunctions in fixed point theory and related topics, J. Math. Anal. Appl. 97 (1983), 151-201.

13. _ Fixed points for Kakutani factorizable multifunctions, J. Math. Anal. Appl. 152 (1990), 46-60.

14. Fixed Point Theory and Applications (M. A. Théra and J. B. Baillon, eds.), Longman Sci. Tech., Essex, 1991, pp. 293-302.

15. S. Park, Fixed point theorems on compact convex sets in topological vector spaces, Contemp. Math., vol. 72, Amer. Math. Soc., Providence, RI, 1988, pp. 183-191.

16. Some coincidence theorems on acyclic multifunctions and applications to KKM theory, Proc. 2nd Int. Conf. on Fixed Point Theory and Applications (Halifax, June 9-14, 1991) (K.-K. Tan, ed.), World Scientific, River Edge, NJ, 1992, pp. 248-277.

17. __ Fixed point theory of multifunctions in topological vector spaces, J. Korean Math. Soc. 29 (1992), 191-208.

18. _ Cyclic coincidence theorems for acyclic multifunctions on convex spaces, J. Korean Math. Soc. 29 (1992), 333-339.

19. M. J. Powers, Lefschetz fixed point theorems for a new class of multi-valued maps, Pacific J. Math. 42 (1972), 211-220.

20. S. Reich, Approximate selections, best approximations, fixed points, and invariant sets, J. Math. Anal. Appl. 62 (1978), 104-113. 
21. _ Fixed point theorems for set-valued mappings, J. Math. Anal. Appl. 69 (1978), 353-358.

22. I. Singer, Some remarks on approximative compactness, Rev. Roumaine Math. Pures Appl. 9 (1964), 167-177.

(S. Park) Department of Mathematics, Seoul National University, Seoul 151, Korea E-mail address: shpark@math.snu.ac.kr

(S. P. Singh and B. Watson) Department of Mathematics, Memorial University of Newfoundland, St. John's Newfoundland, CaNada A1C 5S7

E-mail address, S. P. Singh: spsingh@kean.ucs.mun.ca 